\title{
INVENTARIO DE LOS RECURSOS HUMANOS Y MATERIALES IMPLICADOS EN LA ATENCIÓN SANITARIA BUCODENTAL PÚBLICA EN LA COMUNIDAD VALENCIANA
}

\author{
${ }^{1}$ María Paulina Pieschacón Gutiérrez, ${ }^{2}$ José Manuel Almerich Silla. \\ ${ }^{1}$ Odontóloga U. Santo Tomás, Especialista Salud Pública U. de Chile, DEA en Economía y Gestión en Salud U. Politécnica de Valencia. \\ DEA en Estomatología y aspirante a Doctor en Odontología Universitat de València - España. ${ }^{2}$ Profesor Titular de Odontología \\ Preventiva y Comunitaria, Director del Departamento de Estomatología Universitat de València - España.
}

Autor responsable de correspondencia: María Paulina Pieschacón $G$.

Correo electrónico: palipies@yahoo.com

\begin{abstract}
RESUMEN
Objetivo: Realizar un inventario de los recursos públicos en la atención bucodental de la Comunidad Valenciana (España). Materiales y métodos: Se realizó un estudio descriptivo de los recursos humanos y materiales implicados en la atención bucodental pública. Se visitaron los hospitales, centros de atención primaria y centros de especialidades de los 22 departamentos de salud, se realizaron entrevistas estructuradas al personal.

Resultados: De los 120 centros de atención, 99 fueron de atención primaria, seguidos de especialidades y hospitales. Todos reportaron instrumental de examen y de cirugía. No se observo instrumental y material para operatoria, endodoncia y periodoncía, en los diferentes centros de atención. La existencia de equipamiento fue similar para todos, se percibió una buena dotación con equipos informáticos en la mayoría de ellos. Se destacó también adecuadas plantas físicas y consultas dentales.

Conclusiones: Por primera vez, se hizo un inventario de los recursos humanos y materiales destinados a la atención bucodental pública, que reflejó la existencia de un número importante de profesionales pero insuficiente para atender con las nuevas prestaciones la población infantil de la Comunidad Valenciana. En general se percibió buena dotación; sin embargo, se requieren más recursos humanos, materiales e instrumental apropiado para tratar la caries dental, la enfermedad periodontal y la patología pulpar. [Pieschacón MP, Almerich JM. Inventario de los recursos humanos y materiales implicados en la atención sanitaria bucodental pública en la Comunidad Valenciana. Ustasalud 2009; 8: 91 - 100]
\end{abstract}

Palabras clave: Recursos públicos, Atención en salud oral, Salud pública, Comunidad Valenciana.

\section{INVENTORY OF HUMAN AND DENTAL MATERIAL RESOURCES IN THE PUBLIC HEALTH SERVICE IN VALENCIAN COMMUNITY}

\begin{abstract}
Objective: To prepare an inventory of public resources for oral health care in the Autonomous Valencian Community (Spain). Methods: A descriptive study of human and dental material resources involved in public oral health service was done. The hospitals, primary care and specialty centers of the 22 health departments were visited and structured interviews were conducted for staff. Results: Of the 120 centers, 99 were primary care, followed by specialties and hospitals. There were instrumental for examination and surgery but there were not for surgical, endodontic and periodontal procedures at many health care centres. The existence of equipment was similar for all, a good supply was perceived to computers in most of them. It was also noted good physical and dental facilities.

Conclusions: For the first time, it was done an inventory of human and material resources for public dental care, reflecting the existence of a significant number of professionals, but insufficient to meet the new benefits for the children of the Valencian Community. It was generally felt good supply, however, requires more human resources, materials and proper equipment for the treatment dental caries, periodontal and pulpal disease.
\end{abstract}

Key words: Public resources, Health dental care, Public health, Valencian Community.

Recibido para publicación: 5 de octubre de 2009. Aceptado para publicación: 20 de diciembre de 2009. 


\section{INTRODUCCIÓN}

La misión de los servicios de salud es contribuir a mejorar la salud del individuo y de la sociedad. Sin embargo, situaciones como la complejidad de las prestaciones, la organización de los servicios, los recursos limitados, las presiones sociales y la demanda de los servicios, entre otros, se convierten en retos que se tienen que asumir para que los servicios lleguen de manera eficiente y equitativa a la población o a grupos de población más necesitada; esto quiere decir, mejorar la salud a partir de la utilización adecuada de los recursos disponibles y aumentar la igualdad de oportunidad para obtener acceso a la atención sanitaria.

En España, la mayor parte de la asistencia bucodental a la población se realiza a través del sistema privado, las prestaciones del sector público en adultos se limitan al diagnóstico y al alivio del dolor mediante la extracción dentaria. ${ }^{1}$ En la población infantil y adolescente se amplía con programas educativos, preventivos instaurados a partir de los años 80 , algunos con prestaciones de odontología restauradora en los 90 y otros con programas incrementales después del año 2002. ${ }^{2}$ A lo largo de este tiempo se han creado nuevas leyes como la $14 / 86$, Ley General de la Sanidad, el Real Decreto 63/1995, ${ }^{3}$ sobre las prestaciones sanitarias del Sistema Nacional de Salud (SNS), la ley 16/2003, sobre Cohesión y Calidad del SNS y se regula la profesión de odontólogo, protésico e higienista dental a través de la ley 10/1986, y del Real Decreto 1594/1994. ${ }^{4}$ Además, están el Real decreto 1030/2006, ${ }^{5}$ que actualiza y amplía las prestaciones sanitarias del SNS y el Real Decreto 111/2008 que regula la concesión de subvenciones a las Comunidades Autónomas para la promoción de actividades para la salud bucodental infantil durante el 2008 con posibilidad de ampliarse hasta el año $2015 .^{6}$

Con la ley de cohesión y calidad del Sistema Nacional de Salud que otorga a las Comunidades Autónomas el reconocimiento de las prestaciones dentales, se produce un cambio sustancial en el sistema de provisión de servicios dentales a la población. La transferencia de las competencias en sanidad a las comunidades autónomas, otorgó a los gobiernos regionales la mayor parte de la planificación y organización de los servicios sanitarios y por ende los programas de atención buco-dental, los cuales, se han desarrollado en las distintas regiones. ${ }^{?}$

Con la transferencia de competencias en materia de salud a las Comunidades Autónomas, se crearon diversos programas de salud bucodental que implementaban enjuagues fluorados y actividades educativas en el ámbito escolar. Posteriormente con los resultados de los estudios epidemiológicos nacionales y locales, se conoció la situación de la enfermedad bucodental en la población, lo que generó que, después de los años 90 , se comenzara a incluir la odontología restauradora en el sector público. ${ }^{1}$

Desde aquellos años hasta el momento actual los servicios dentales públicos han experimentado un enorme crecimiento, generalmente en lo referido a la población infantil y adolescente. Más de la mitad de las Comunidades Autónomas disponen de líneas estratégicas enmarcadas en sus programas de salud bucodental, pero con esfuerzos y resultados dispares entre ellas. En lo que respecta a las prestaciones infantiles, algunas comunidades, entre las que se destacan el País Vasco, Navarra, Andalucía y Murcia garantizan a través del Plan de Atención Dental Infantil (PADI) la atención de las necesidades preventivas y reparadoras de los niños, mediante una amplia red de consultas concertadas por capitación..$^{8-12}$ Otras comunidades, han tomado ya dicho modelo con algunas modificaciones. ${ }^{1,2}$

En el país se tienen instaurados tres modelos de servicios bucodentales para la población infantil y juvenil, además de la odontología de cupo (urgencias) para la población adulta. Cada comunidad autónoma define la financiación, la provisión de servicios y las prestaciones en cada uno de los siguientes modelos: ${ }^{2}$

\section{Modelo público}

Son los servicios de la red pública de Atención Primaria prestados por odontólogos asalariados, se rigen por el Real Decreto 1030/2006. Las comunidades que presentan este modelo son Asturias, Baleares, Canarias, Galicia, Comunidad de Madrid, La Rioja, Comunidad Valenciana, Ceuta y Melilla. Cataluña y Cantabria tienen sus propios programas apoyados en el mismo decreto.

\section{Modelo mixto, público y privado}

Tienen su propia legislación y cartera de servicios. Los tratamientos básicos los hace la red pública y los tratamientos especializados se remiten a la red privada cuyo pago concertado es por acto médico. A este modelo pertenecen las comunidades Castilla-La Mancha, y Castilla y León.

\section{Modelo Programa de Asistencia Dental Infan- til (PADI)}

Está caracterizado por la financiación pública, la provisión pública y privada, y la libre elección. El pago a los privados es por capitación para la patología general y por acto medico, a tarifa concertada, para otros tratamientos. Ambos sectores realizan los mismos tratamientos preventivos y restaurado- 
res. Este modelo lo inicio el País Vasco y Navarra en los años 90, posteriormente lo desarrollaron Andalucía, Aragón, Extremadura, Murcia y Baleares. ${ }^{1,2,8,9}$

Según datos de la última encuesta epidemiológica, Encuesta Nacional de Salud Oral 2000, en los últimos años se ha producido una mejoría de la salud dental de los españoles. En lo que respecta a la presencia de caries dental por grupos de edad: en los niños de 5 a 6 años cerca del $67 \%$ están libres de caries dental. Este porcentaje desciende al $57 \%$ para el grupo de 12 años, y al 31\% para los adolescentes de 15 años. Sin embargo, para los adultos la prevalencia de caries dental se acerca al $100 \%{ }^{13}$

Con respecto al Índice de Caries en la Dentición Permanente (CAO-D), se observó que a la edad de 12 años fue 1,12 lo que significa niveles muy bajos de caries con base en la clasificación de la Organización Mundial de la Salud (OMS). Es notorio, que la caries es mayor los estratos socioeconómicos bajos que en los altos. A los 15 años el CAO-D asciende a 2,20. Lo contrario sucede en el grupo de 65 a 74 años donde el CAO-D se eleva a 18,1 y mantiene un nivel muy alto para un país desarrollado. ${ }^{13}$

La Comunidad Valenciana ha realizado cuatro estudios que evidencian una prevalencia de caries dental de moderada a baja. ${ }^{14-16}$ En general, se observó una notable evolución en la condiciones de salud bucodental en los niños y adolescentes respecto a las pautas establecidas por la OMS. Sin embargo, también existen estudios que demuestran que la clase social baja y el bajo nivel educacional son factores de riesgo en la distribución de la carie dental $y$, dentro de ella, se puede englobar a la población inmigrante de diferentes etnias y culturas que provienen de países con altos índices de enfermedad bucodental. ${ }^{17-18}$

De acuerdo con la última encuesta de salud oral realizada en la Comunidad Valenciana en el 2004, por Almerich y Montiel, el porcentaje de individuos libres de caries dental fue de $65.8 \%$ a los 6 años, del $51.7 \%$ a los 12 años y desciende hasta un $43.9 \%$ de los 15 a 16 años. En cuanto a la prevalencia de caries por sexo no se encontraron diferencias estadísticamente significativas. ${ }^{16,18}$

Otra publicación reciente sobre el efecto de la inmigración en la prevalencia y experiencia de caries dental en niños de 12 y 15 años de la región de Valencia reveló que la inmigración, el consumo de alimentos cariogénicos, la clase social y la edad eran factores fuertemente asociados con la presencia de caries dental. ${ }^{17}$ Los niños inmigrantes tuvieron más lesiones de caries que los niños españoles; el Índice de Caries Significante $(\mathrm{SiC})$ reportado fue de 3,67 a los 12 años y 6,87 a los 15 años en los niños inmigrantes; en contraste con 2,84 a los 12 años y 4,37 a los 15 años en los niños españoles, diferencias estadísticamente significativas.

El programa de salud bucodental de la Comunidad Valenciana se desarrolló a partir del primer estudio epidemiológico en salud oral realizado en 1986. Su objetivo fue la disminución de la incidencia de caries dental y enfermedad periodontal en la población infantil. Fundamenta sus actividades en los centros docentes, se realiza educación sanitaria, enjuagues con flúor y exámenes orales en el colegio a niños de primero de primaria con la correspondiente remisión a las Unidades de Odontología Preventiva. En los Centros de Atencion Primaria, directamente en la consulta pediátrica con la aplicación del "Protocolo de Actuación Pediátrica para la Promoción de la Salud Bucodental Infantil", y en las Unidades de Odontología Preventiva, con asesoramiento dietético, aprendizaje individualizado de medidas de higiene, selladores de fisuras y aplicación tópica de flúor. $^{19}$

Tanto los resultados positivos que se observan en esta comunidad, como los nuevos retos de disminuir los indicadores y de trabajar con la población inmigrante, plantean panoramas y estrategias para optimizar los recursos y mejorar la asistencia sanitaria. $^{14-18}$

Este último aspecto es muy importante máxime cuando el Ministerio de Sanidad y Consumo, expidió el Real Decreto 111/2008, ${ }^{6}$ por el que se regula la concesión directa de subvenciones a las comunidades autónomas para la promoción de actividades para la salud bucodental infantil durante el 2008. Con la concesión de las subvenciones reguladas se pretende dar cumplimiento a las actividades para la salud bucodental infantil entre los 7 y 8 años de edad durante el año 2008, para extenderse progresivamente a lo largo del tiempo a grupos de edades subsiguientes, hasta alcanzar las edades de 14 y 15 años. Este plan pretende garantizar prestaciones como limpiezas bucales, sellantes, obturaciones, extracciones y revisiones periódicas.

La política anterior deberá estar acorde con Las metas de la salud oral para el año $2020,{ }^{20}$ establecidas conjuntamente por la Federación Dental Internacional (FDI), La OMS, y la International Association for Dental Research (IADR), que incluyen objetivos y focos de aplicación globales con la intención de que cada gobierno haga un análisis de su situación y recursos para establecer metas propias y reales. Entre los objetivos se destaca la necesidad de desarrollar sistemas de salud oral accesibles costo efectivos para la prevención y el control de las enfermedades orales. ${ }^{21}$ 
En este sentido, todos los recursos más el conocimiento, son factores importantes dignos de tener cuenta para cualquier evaluación y mejor utilización de los recursos disponibles. El propósito del presente estudio fue hacer un inventario de los recursos humanos y materiales por departamento de salud, que permitan acorde con los nuevos planteamientos del Ministerio de Sanidad Español, planificar en un posterior estudio un Programa de Salud Bucodental que integre los aspectos preventivos y asistenciales, en la asistencia sanitaria odontológica de los ciudadanos de la Comunidad Valenciana.

\section{MATERIALES Y MÉTODOS}

Se realizó un estudio descriptivo de los recursos humanos y materiales dentales implicados en la atención bucodental de la red pública en la Comunidad Valenciana (Figura 1 y 2 ).

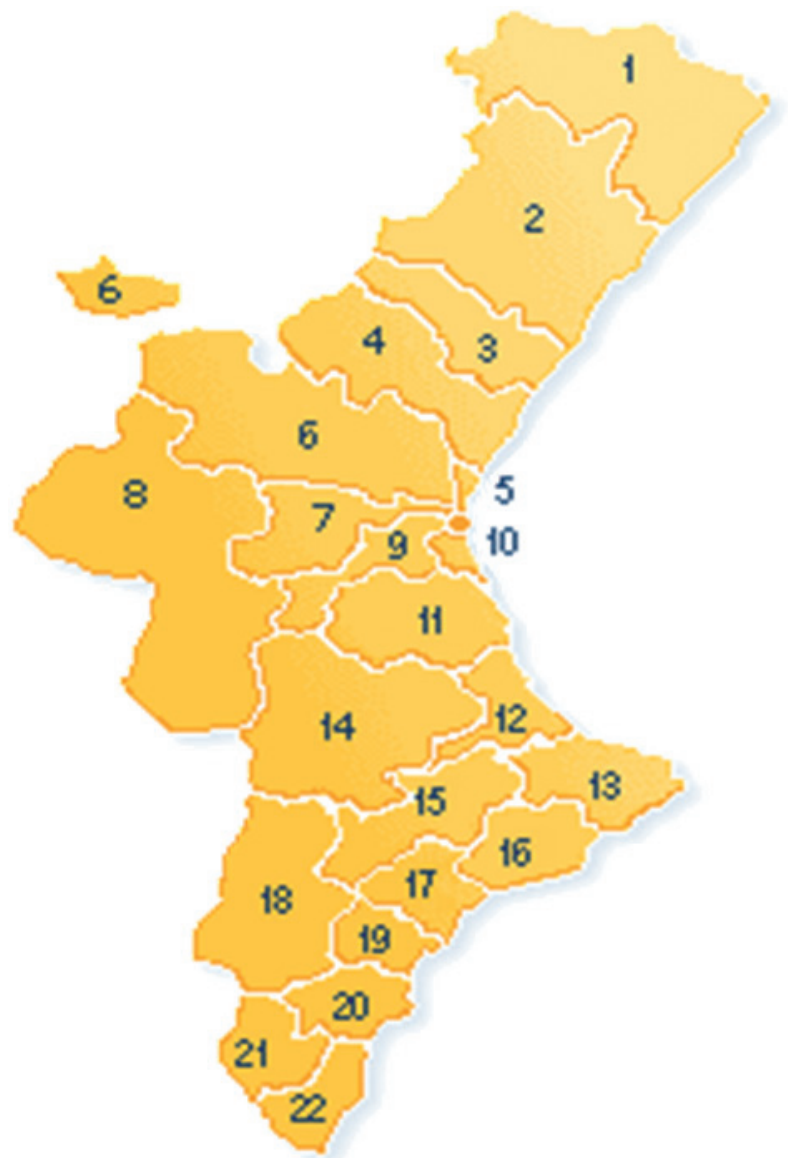

Figura 1. Veintidos departamentos que conforman la Comunidad Valenciana en España

Para la recolección de la información se inició con una revisión sistemática de los recursos disponibles por departamentos a través de los listados disponibles en la Web de la Conselleria de Sanitat y entrevistas telefónicas con los coordinadores de los centros. En el mes de noviembre de 2006, tras obtener el permiso de las direcciones de departamento, se visitaron los hospitales, centros de atención primaria y centros de especialidades de los 22 departamentos de salud, se realizaron entrevistas estructuradas al personal de salud que se encontraba en las instalaciones al momento de la visita. En casos particulares se acudió a los coordinadores médicos y de enfermería.

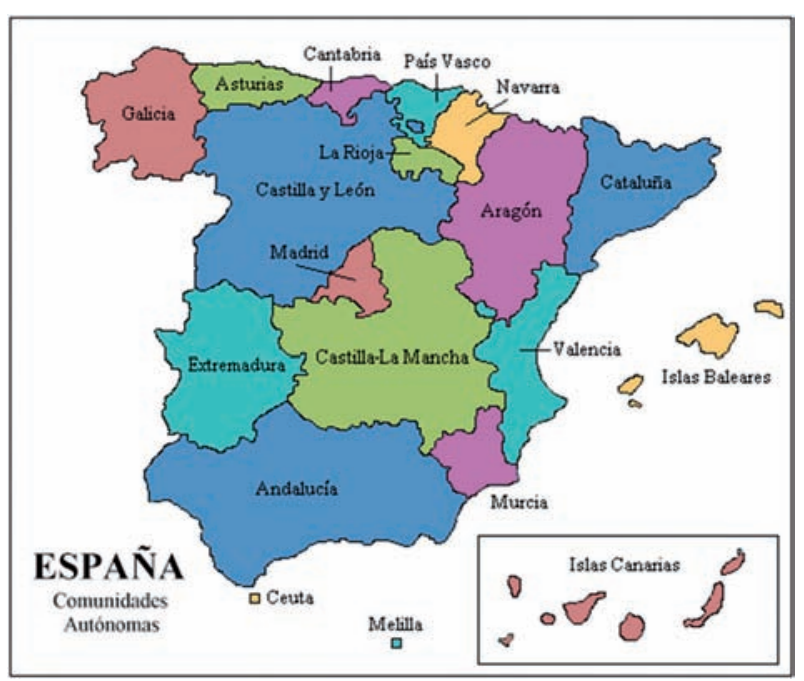

Figura 2. Mapa de la situación geográfica de la Comunidad Valenciana.

Se incluyeron los hospitales, los centros de atención primaria y los servicios de atención especializada de los 22 departamentos pertenecientes a red pública de la Comunidad Valenciana, donde se realizara algún tipo de atención bucodental.

En cuanto a los recursos humanos se tomaron estomatólogos, odontólogos/as, cirujanos maxilofaciales, higienistas, auxiliares y diplomados universitarios en enfermería, vinculados a la atención bucodental pública en todos los departamentos seleccionados.

Los recursos materiales se analizaron de acuerdo a si se tiene o no, sillones, la utilización diaria de los sillones, unidades de $\mathrm{Rx}$, instrumental para diferentes procedimientos, materiales dentales para diferentes procedimientos y otros equipos de apoyo y, si utilizan o no protocolos de atención.

En total se diligenciaron 124 registros con la información. Se tomaron como variables para estudiar los recursos humanos y materiales implicados en la atención bucodental (Tabla 1).

\section{RESULTADOS}

La Comunidad Valenciana está dividida en 22 departamentos de salud, para el estudio se analizaron 
120 centros de atención. Sólo en siete departamentos se prestaba atención dental de nivel hospitalario. Los Centros de Atención Primaria son los centros de atención más frecuentemente observados en todos los departamentos para un total de 99.

El número más elevado de profesionales de la salud oral se observó para los estomatólogos- odontólogos e higienistas. Por departamentos hay mayor número de cirujanos maxilofaciales en el departamento 7 y 17. Sin embargo, no se prestaba atención en cirugía maxilofacial en la mayoría de los departamentos y el servicio debía remitirse a los hospitales. El número más alto de estomatólogos se observó en el departamento 9 y 11, el de odontólogos en los departamentos 20 y 22 . El número más alto de higienistas estaba en los departamentos 10 y 20. La contratación de las auxiliares y Diplomados Universitarios de Enfermería (DUE) fue bastante baja en todos los departamentos.

Aún cuando se encuentran equipos radiográficos intraorales en todos los departamentos, hay muchos centros que carecen de ellos. Sólo en los departamentos 3, 14 y 22 hay uno por consulta. Los de peor relación son el 9, 16 y 12. Los ortopantógrafos se en- contraban en baja cantidad, generalmente, ubicados en los hospitales. Todos los centros reportaban tener instrumental de examen, seguido del instrumental de cirugía. El instrumental para operatoria, endodoncia y periodoncía, prácticamente no existía en los diferentes centros de atención de los departamentos.

Todos los centros de atención reportaron existencia de materiales para prevención (UOPs) y para exodoncias dentales (CCEE-Hospitales). En términos generales, no había existencia de materiales dentales para realizar tratamientos de operatoria, endodoncia y periodoncía en ninguno de los centros de atención de los departamentos.

La existencia de equipamiento es muy similar entre los diferentes centros y departamentos y, en general, se percibió una buena dotación; es importante anotar que en algunos departamentos no había piezas de alta velocidad, de baja velocidad y de limpieza mecánica. Fue notoria la existencia de equipos informáticos en la mayoría de los centros. Se destaca también adecuadas plantas físicas y consultas dentales en los diferentes centros de la Comunidad Valenciana (Tabla 2).

Tabla 1. Variables del estudio

\section{VARIABLES DE ESTUDIO}

\begin{tabular}{|c|c|c|}
\hline Departamento de Salud & Número de sillones & Materiales de prevención \\
\hline Hospital & Número de rayos $\mathrm{X}$ & Materiales de operatoria \\
\hline Centro de Salud & Instrumental de examen & Materiales de cirugía \\
\hline Centro de Atención Especializada & Instrumental de operatoria & Materiales de endodoncia \\
\hline Cirujano maxilofacial & Instrumental de cirugía & Materiales de periodoncia \\
\hline Estomatólogo & Instrumental de endodoncia & Lámpara de fotocurado \\
\hline Odontólogo & Instrumental de periodoncia & Equipos de esterilización \\
\hline Higienista & & Turbina de alta velocidad \\
\hline Diplomados de enfermería (DUE) & & Turbina de baja velocidad \\
\hline Auxiliar de consultorio & $\begin{array}{l}\text { Días de ocupación de los sillones (lunes, } \\
\text { martes, miércoles, jueves y viernes) y } \\
\text { jornadas. }\end{array}$ & Aspiración quirúrgica \\
\hline \multicolumn{3}{|l|}{ Sistemas informáticos } \\
\hline & & Aparatos de limpieza mecánica \\
\hline
\end{tabular}


Del total de la población, se tomaron como referencia las personas entre los 15 años hasta mayores de 85 años como población asignada a hospitales y centros de atención especializada (CCEE) en cada departamento. Los departamentos 9,10 y 22 tenían la mayor población. Llama la atención el escaso número de profesionales para la atención de los adultos en todos los departamentos y en especial aquellos con mayor número de personas (Tabla 2).

En general, no se observó una distribución equilibrada de los profesionales (estomatólogos-odontólogos e higienistas), quienes se encontraban en baja cantidad con respecto a la población asignada en la mayoría de Unidades de Odontología Preventiva (UOP). Los higienistas fueron el recurso humano más numeroso en este tipo de servicio, seguido por los odontólogos. Muy pocas auxiliares de consultorio se encontraban asignadas a las UOPs. El departamento con mejor ratio poblacional es el 8 seguido por el 3 y el 1; el que cuenta con mayor recurso humano fue el 20 , el de peor relación, es decir, mayor población infantil asignada y un número bajo de recursos humanos fue el 22 (Tabla 3, Figura 3).

Tabla 2. Relación de profesionales en Hospitales y CCEE con la población asignada para cada departamento de salud en la Comunidad Valenciana

\begin{tabular}{|c|c|c|c|c|c|c|c|}
\hline \multirow{2}{*}{ DPTO } & \multicolumn{3}{|c|}{ HOSPITAL } & \multicolumn{3}{|c|}{ CCEE } & \multirow{2}{*}{$\begin{array}{c}\text { POBLACIÓN } \\
15>85 \text { años }\end{array}$} \\
\hline & SILLÓN & MAXILOS & ESTOMAT. & SILLÓN & ESTOMAT. & ODONTOL. & \\
\hline 1 & 0 & 0 & 0 & 0 & 0 & 0 & 79.700 \\
\hline 2 & 2 & 3 & 0 & 1 & 4 & 1 & 244.554 \\
\hline 3 & 0 & 0 & 0 & 0 & 0 & 0 & 155.985 \\
\hline 4 & 0 & 0 & 0 & 1 & 0 & 2 & 97.361 \\
\hline 5 & 0 & 0 & 0 & 2 & 4 & 0 & 298.943 \\
\hline 6 & 0 & 0 & 0 & 0 & 0 & 0 & 258.381 \\
\hline 7 & 1 & 9 & 0 & 1 & 4 & 0 & 299.759 \\
\hline 8 & 0 & 0 & 0 & 0 & 0 & 0 & 44.660 \\
\hline 9 & 5 & 2 & 5 & 3 & 5 & 1 & 323.628 \\
\hline 10 & 2 & 0 & 2 & 2 & 5 & 0 & 337.464 \\
\hline 11 & 0 & 0 & 0 & 0 & 0 & 0 & 223.802 \\
\hline 12 & 1 & 0 & 0 & 0 & 2 & 0 & 158.000 \\
\hline 13 & 0 & 0 & 0 & 1 & 0 & 2 & 158.745 \\
\hline 14 & 0 & 0 & 0 & 2 & 2 & 0 & 172.471 \\
\hline 15 & 0 & 0 & 0 & 1 & 1 & 0 & 121.224 \\
\hline 16 & 0 & 0 & 0 & 1 & 2 & 0 & 187.118 \\
\hline 17 & 5 & 9 & 0 & 2 & 2 & 0 & 198.951 \\
\hline 18 & 0 & 0 & 0 & 1 & 2 & 0 & 185.981 \\
\hline 19 & 0 & 0 & 0 & 0 & 4 & 0 & 222.197 \\
\hline 20 & 0 & 0 & 0 & 1 & 1 & 1 & 244.637 \\
\hline 21 & 0 & 0 & 0 & 0 & 0 & 0 & 292.370 \\
\hline 22 & 1 & 2 & 0 & 0.5 & 1 & 3 & 306.659 \\
\hline
\end{tabular}

Datos población: Informe de indicadores demográficos en SIP (2007/01). ${ }^{21}$ 
Tabla 3. Ratios de población de 5 a 14 años por odontólogos e higienistas en los Centros de Salud (UOP) para cada departamento de salud en la Comunidad Valenciana

\begin{tabular}{|c|c|c|c|c|c|}
\hline \multirow{2}{*}{ DPTO } & \multirow{2}{*}{$\begin{array}{l}\text { POBLACIÓN } \\
5 \text { a } 14 \text { años }\end{array}$} & \multicolumn{4}{|c|}{ CENTRO DE SALUD (UOP) } \\
\hline & & ODON-EST & RATIO POBL & HIGUIENIST & RATIO POBL \\
\hline 1 & 9.337 & 2 & 4.665 & 2 & 4.665 \\
\hline 2 & 31.936 & 3 & 10.645 & 3 & 10.645 \\
\hline 3 & 20.825 & 5 & 4.165 & 3 & 6.941 \\
\hline 4 & 11.348 & 1 & 11.348 & 1 & 11.348 \\
\hline 5 & 35.134 & 1 & 35.134 & 2 & 17.576 \\
\hline 6 & 36.176 & 3 & 12.058 & 3 & 12.058 \\
\hline 7 & 34.129 & 2 & 17.064 & 5 & 6.825 \\
\hline 8 & 5.142 & 2 & 2.571 & 1 & 5.142 \\
\hline 9 & 40.808 & 2 & 20.404 & 1 & 40.808 \\
\hline 10 & 38.652 & 2 & 19.326 & 7 & 5.521 \\
\hline 11 & 28.292 & 2 & 14.146 & 2 & 14.146 \\
\hline 12 & 20.638 & 1 & 20.638 & 1 & 20.638 \\
\hline 13 & 17.869 & 1 & 17.869 & 2 & 8.934 \\
\hline 14 & 22.699 & 2 & 11.349 & 3 & 7.566 \\
\hline 15 & 15.027 & 3 & 5.009 & 3 & 5.009 \\
\hline 16 & 19.730 & 3 & 6.576 & 4 & 4.932 \\
\hline 17 & 25.514 & 2 & 12.757 & 4 & 6.378 \\
\hline 18 & 25.500 & 2 & 17.750 & 4 & 6.375 \\
\hline 19 & 29.000 & 3 & 9.666 & 4 & 7.250 \\
\hline 20 & 32.566 & 4 & 8.141 & 7 & 4.652 \\
\hline 21 & 38.042 & 2 & 19.021 & 3 & 12.680 \\
\hline 22 & 39.283 & 1 & 39.283 & 1 & 39.283 \\
\hline
\end{tabular}

Datos población: Informe de indicadores demográficos en SIP $(2007 / 01){ }^{21}$

La Figura 4 muestra el porcentaje de utilización (ocupación) de los sillones dentales por día para cada departamento. El bajo porcentaje de utilización en la mayoría de los departamento se debe a que la atención dental es predominante en la jornada de la mañana, muy poca en ambas jornadas y casi ninguna en las tardes (Figura 4). 


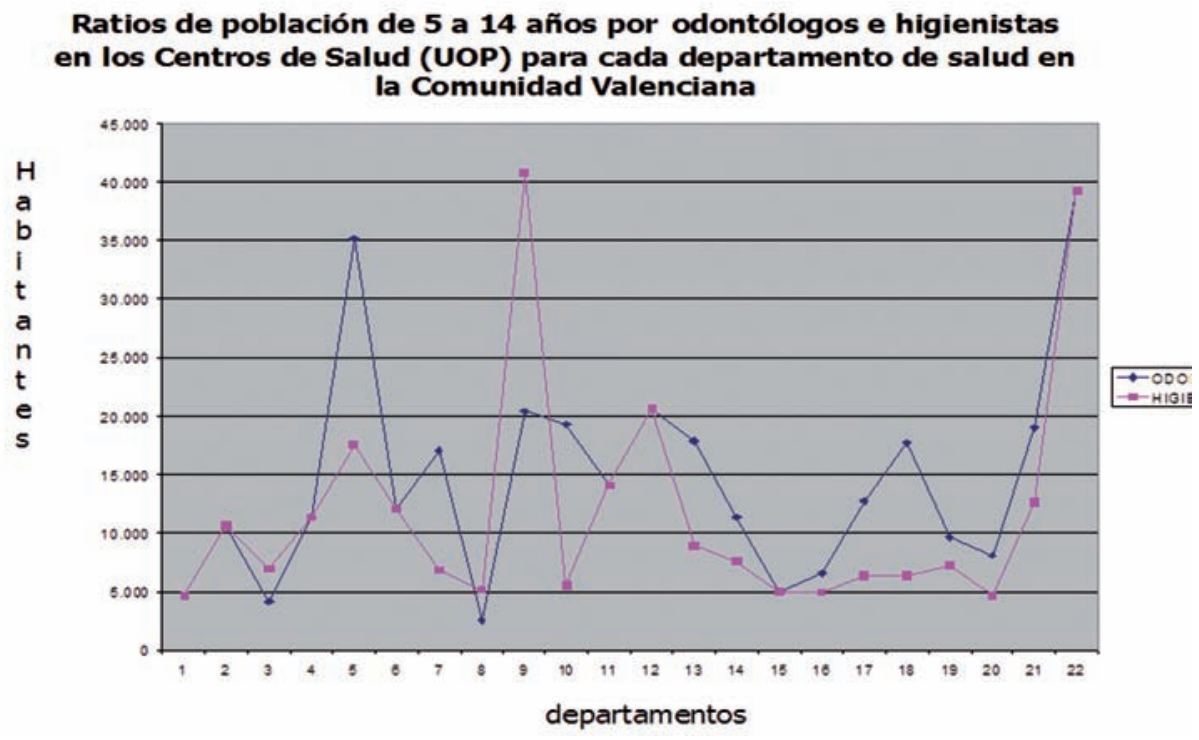

Figura 3. Relación de la población de 5 a 14 años por odontólogo e higienista en las UOP.

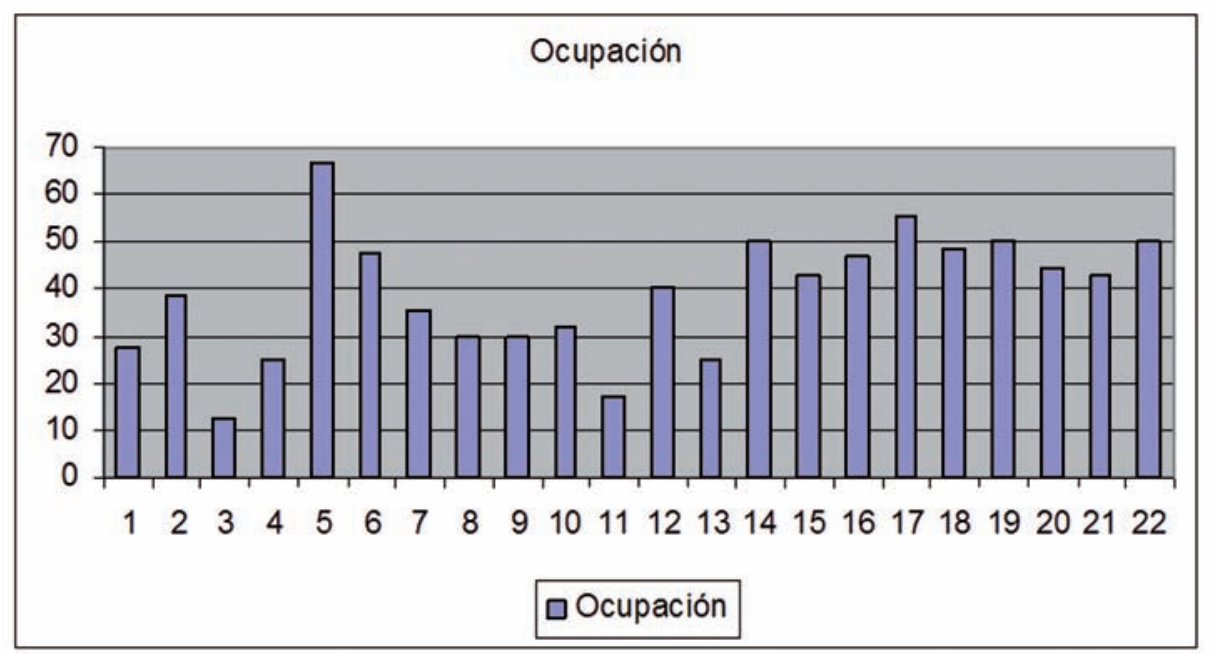

Figura 4. Porcentaje de ocupación de sillones dentales por días y jornadas según el departamento

\section{DISCUSIÓN}

El estudio tomo 22 departamentos y 120 establecimientos de salud clasificados como Centros de Atención Primaria donde se encuentran las Unidades de Odontología Preventiva (UOP), Centros de Especialidades (CCEE) y Hospitales, ubicados en la Comunidad Valenciana.

La Comunidad Valenciana ha desarrollado desde 1986, un programa de salud oral, con el objetivo, de disminuir la incidencia de caries dental y enfermedad periodontal en la población infantil; ${ }^{16}$ fundamenta sus actividades en los centros docentes, se realiza educación sanitaria, enjuagues con flúor y examen oral en el colegio a los niños de primero de primaria con la correspondiente remisión a las diferentes Unidades de Odontología Preventiva
(UOP). Este programa ha tenido continuidad lo que ha permitido atribuirle los buenos resultados en los indicadores de salud oral mostrados en los estudios epidemiológicos. ${ }^{14-16,18}$

A pesar de lo anterior, existe una necesidad potencial de atención odontológica, según el último Estudio de Salud Bucodental Infantil en la Comunidad Valenciana 2004, ${ }^{16}$ el índice de morbilidad dental Índice de Caries en la Dentición Permanente (componente cariado) ha aumentado del $52.7 \%$ al $65.5 \%$, es decir, ha aumentado el número de caries no tratadas en niños. Estos datos son muy superiores al índice general en España que es del $44.6 \%$ y al de Navarra que es del $21 \% .^{13}$ Igualmente, el índice de restauración (componente obturado), ha disminuido del $45.3 \%$ al $32.7 \%$ en la Comunidad Valenciana, 
esto contrasta con la calidad de los recursos físicos y humanos presentes, pudiéndose realizar tratamientos restauradores como sucede en la mayoría de comunidades autónomas que tienen programas de atención odontológica infantil ya instaurados..$^{8-11}$

Respecto a la inmigración, la población de origen extranjero analizada según el último estudio de salud bucodental en la comunidad infantil valenciana, representa el 7.6\% del total en la edad de los 6 años, el 6\% a los 12 años y el 6.4\% a los 15-16 años, dicha población muestra unos índices COP-D superiores a los obtenidos para los niños españoles. ${ }^{16-18}$ $\mathrm{Al}$ aumentar la población inmigrante aumentan los niveles de caries dental, lo que puede estancar o modificar la prevalencia de caries en las edades de 6,12 y 15 años.

La Comunidad Valenciana tiene también la odontología de cupo (urgencias), la cual se encarga de la atención al dolor y las exodoncias, este tipo de atención se realiza en los Centros de Especialidades. Cuando se requiere realizar remisiones para tratamientos quirúrgicos más complejos, se realizan por estomatólogos o cirujanos maxilofaciales en algunos hospitales. En la Comunidad Valenciana hay 18 Centros de Especialidades y en siete departamentos hay servicio de cirugía maxilofacial en los hospitales.

En la publicación Servicios Públicos de Atención Bucodental en España, SESPO/2005, ${ }^{1}$ las comunidades mejor integradas en odontología restauradora son Andalucía, Aragón, las dos Castillas, Extremadura, Murcia, Navarra, y el País Vasco que cubre todo tipo de tratamiento restaurador. En las demás, hay variaciones y las prestaciones se reducen a obturaciones en molares y no de forma generalizada. En la Comunidad Valenciana, se realizan las prestaciones del Programa de Salud Bucodental, ${ }^{19}$ predominantemente preventivas, junto con algunas acciones restauradoras en el Servicio de Estomatología del Hospital General, en el que se realizan tratamiento de la caries a los escolares. ${ }^{20}$

En el estudio se observó que existe buena infraestructura física, pero se carece de instrumental y materiales para realizar otros procedimientos restauradores como tratamiento de la caries dental, afecciones de la encía y la pulpa, lo que difiere con la necesidad de tratamiento en niños y principalmente en adultos que presentan una dramática pérdida de dientes y una carencia importante de tratamiento. ${ }^{1,2,9,13,16,17}$

Igualmente, hay pocos aparatos de radiografía extraoral (ortopantógrafos) en la Comunidad Valenciana; por lo tanto, los pacientes que son atendidos en los diferentes centros de especialidades y que necesitan esta ayuda diagnóstica, se tienen que desplazar en la mayoría de casos, para la toma de estas radiografías, o, pagar estos exámenes a costos más elevados en centros privados. Los aparatos para radiografías periapicales ubicados en las UOPs se encuentran muchas veces subutilizados, en algunas ocasiones por falta de equipos de revelado y en otras por falta de licencias de radioproteción.

Respecto a los recursos humanos, según la Encuesta de Salud Oral en España (2000), ${ }^{13}$ se requiere un promedio de tiempo de asistencia de 25 a 30 minutos por persona y año, y un cociente de odontólogos/habitantes al año 2000 de 1 odontólogo por cada 2.279 habitantes. De acuerdo con el Informe de Indicadores Demográficos que se encuentra en el Sistema de Información Población (SIP) periodo 2007/01, llama la atención el gran número de personas vinculadas al sistema en cada uno de los departamentos. ${ }^{21}$ En contraste, el estudio reveló que existe un número bajo de profesionales para la población asignada, así el número total de estomatólogos y odontólogos es 105 , de cirujanos maxilofaciales 25 , de higienistas 68, auxiliares 26 y DUEs 13, muy por debajo de la relación de habitantes tal como lo muestran los datos de los resultados. Al tomar como referencia las UOPs y la población de 5 a 14 años las ratios poblacionales son muy altas.

Esta situación coincide con las afirmaciones de Pinilla sobre la escasa oferta de prestaciones odontológicas en el sistema público español, en donde la mayoría del recurso humano existente trabaja en el sector privado y la red pública apenas recoge el $10 \%$ del total de profesionales del sector. ${ }^{22}$ Comparado con otros países de la Unión Europea, muestra cómo en Suecia o Finlandia el 50\% de los odontólogos ejerce en el servicio público dental, al igual que en Dinamarca o Irlanda. En el Reino Unido, 20\% son asalariados de hospitales o centros ambulatorios y otros $70 \%$ son odontólogos de cabecera. ${ }^{23,24}$

Todos los programas de salud oral deben evaluarse, por lo tanto, se necesita incluir información relacionada con la fuerza de trabajo, número, necesidades y demanda. La evaluación de la eficiencia de las políticas en salud oral se fundamenta en parte en la accesibilidad a los servicios por parte de la población en general; es necesario tratar de enfocar las acciones hacía los grupos poblacionales de jóvenes, personas mayores y otros catalogados como grupos de riesgo. ${ }^{25-27}$

En este sentido las personas, el tiempo, las instalaciones, el equipamiento y el conocimiento son factores importantes para tener en cuenta en cualquier evaluación y mejor utilización de los recursos dispo- 
nibles. ${ }^{26}$ La importancia de conocer estos recursos en la atención pública, radica en la posibilidad de realizar modelos de evaluación económica y crear mejores métodos administrativos y económicos, es decir, posibilita una mejor organización para la prestación de los servicios de atención sanitaria. Lo anterior es relevante con la entrada en vigor de los Reales Decretos 111/2008 y 1462/2009, que pretenden poner fin a la falta de equidad del servicio odontológico para los escolares a nivel público, lo que garantiza la financiación de las actividades preventivas y curativas en todo el territorio español hasta el año 2015. ${ }^{6,28}$

Uno de los principales retos de los programas de salud oral, es que para el futuro próximo puedan transformar los conocimientos y las experiencias de prevención y atención de la enfermedad en programas efectivos y mejorar las alternativas de atención de las personas vinculadas a los sistemas de salud en Europa, particularmente de aquellos catalogados como poblaciones vulnerables..$^{21,25,26}$

\section{BIBLIOGRAFÍA}

1. Cortés FJ, Cerviño S, Casals E. Servicios Públicos de Salud Bucodental en España. Legislación y cartera de servicios en las CC.AA 2005. 2nda. Edición. Barcelona: SESPO; 2005. URL disponible en: http://www.infomed.es/sespo/ libro_legislacion.pdf

2. Cortés FJ y colaboradores. Servicios Públicos de Salud Bucodental en España. 3ra. edición. SESPO, 2008. URL disponible en: http://www.infomed.es/sespo/invierno08/ Ponencias_SESPO_Barcelona_2008_sin_tapas.pdf

3. Real Decreto 63/1995. Asistencia Sanitaria de la Seguridad Social. Ministerio de Sanidad y Consumo. Gobierno de España. BOE 10/2/1995, núm. 35

4. Real Decreto 1594/1994. Regula la profesión de Odontólogo, Protésico dental e Higienista dental. Ministerio de Sanidad y Consumo. Gobierno de España. BOE 8/9/1994, núm. 215.

5. Real Decreto $1030 / 2006$. Se establece la cartera de servicios comunes del sistema nacional de Salud y el procedimiento para su actualización. BOE16/09/2006, núm. 222.

6. Real Decreto 111/2008. Ministerio de Sanidad y Consumo. Gobierno de España, 2008.

7. Informe Anual del Sistema Nacional de Salud. Ministerio de Sanidad y Consumo. España, 2003.

8. Simón F. Evauación de los seis primeros años de desarrollo del Programa de Asistencia Dental Infantil (PADI) de la comunidad Autónoma del País Vasco. (1990-1995) [Tesis Doctoral]. Madrid: Universidad Complutense de Madrid; 1996.

9. Freire JM. El Programa Dental de Atención Infantil (PADI) de Navarra y del País Vasco: logros y nuevas metas. An Sist Sanit Navar 2004.

10. Cortés JM, Ramón E, Cuenca. Doce años de programa de asistencia dental infantil (PADI) en Navarra (1991-2002). Utilización e indicadores de salud. An Sist Sanit Navar 2003; 26.

11. Promoción de la Salud Bucodental en el ámbito escolar: Programa Aprende a Sonreír. Junta de Andalucía: Consejería de Salud. Sevilla, 2003.
12. Servicio Murciano de Salud. Programa de salud bucodental infantil. URL disponible en: www.murciasalud.es

13. Llodra JC, Bravo M, Cortés FJ. Encuesta de Salud Oral en España (2000). RCOE 2002; 7: 19 - 63.

14. Zurriaga O, Ibáñez J. La Salud Bucodental en la Comunidad Valenciana. Encuesta de prevalencia en la población infantil. Monografias Sanitarias, Serie A (estudios). No. 9. Valencia: Conselleria de Sanitat i Consum. Generalitat Valenciana; 1987.

15. Estudio de Salud Bucodental en la Comunidad Valenciana 1998. Informes de Salud No 47. Dirección General de Salud Pública. Generalitat Valenciana. 1999.

16. Estudio de Salud Bucodental en la Comunidad Valenciana 2004. Informes de Salud No 81. Dirección General de Salud Pública. Generalitat Valenciana. 2005.

17. Almerich-Silla JM, Montiel-Company JM. Influence of immigration and other factors on caries in 12- and 15-yrold-children. Eur J Oral Sci 2007; 115: 378 - 383.

18. Almerich-Silla JM, Montiel-Company, JM. Oral health survey of the child population in the Valencia Region of Spain (2004). Med Oral Patol Oral Cir Bucal 2006; 11: E369 - E381.

19. Programa de Salud Bucodental. Guía del profesorado. Conselleria de Sanitat. Valencia, España. 2004.

20. Poveda R, Jiménez Y, Gavalda C, Sanchis JM, Carbonell E, Margaix M, Sarrión G. Pedriatric dental care in a tertiary public hospital. Four years of experience in the Service of Stomatology of Valencia University General Hospital (Valencia, Spain). Med Oral Patol Oral Cir Bucal 2008; 13: E331- E335.

21. Informe de Indicadores Demográficos en el Sistema de Información Poblacional. Periodo 2007/01. Descriptivo por Centro. Datos suministrados por la oficina de Salud Pública de la Conselleria de Sanidad. Comunidad Valenciana, 2007.

22. Pinilla J. La economía de los servicios de atención bucodental en España. Cuadernos Económicos de I.C.E. No. 67. Ministerio de Industria, Turismo y Comercio. Madrid 2004: 135 - 160

23. Widstrom E, Eaton KA. Oral health systems in Europe Union. Oral Health Prevent Dent 2004; 2: 155 - 194.

24. Community Action Programmer on Health Monitoring European Commission Directorate- General Health Consumer Protection. Selecting Essential Oral Health Indicators in Europe. Report of the Consensus Workshop. University of Granada, Spain. 7-8 May 2004.

25. Hobdell M, Petersen PE, Clarkson J, Johnson N. Global goals for oral health 2020. Int Dent J 2003; 53: $285-288$.

26. Petersen P. The World Oral Health Report 2003. Continuous improvement of oral health in the $21^{\text {st }}$ century-the approach of the WHO Global Oral Health Programmer. WHO 2003.

27. Kaufhold R, Paul Meyer V, Fink D. Comparison of Dental Fees in Europe. The "Euro-Z" Project. The European Journal of Health Economics 2001; 2: $11-17$.

28. Real Decreto 1464/2009. Regula la concesión directa de subvenciones a las comunidades autónomas para la promoción de actividades para la salud bucodental infantil durante el año 2009. Ministerio de Sanidad y Política Social. Gobierno de España. BOE 08/10/2009, núm. 243. 